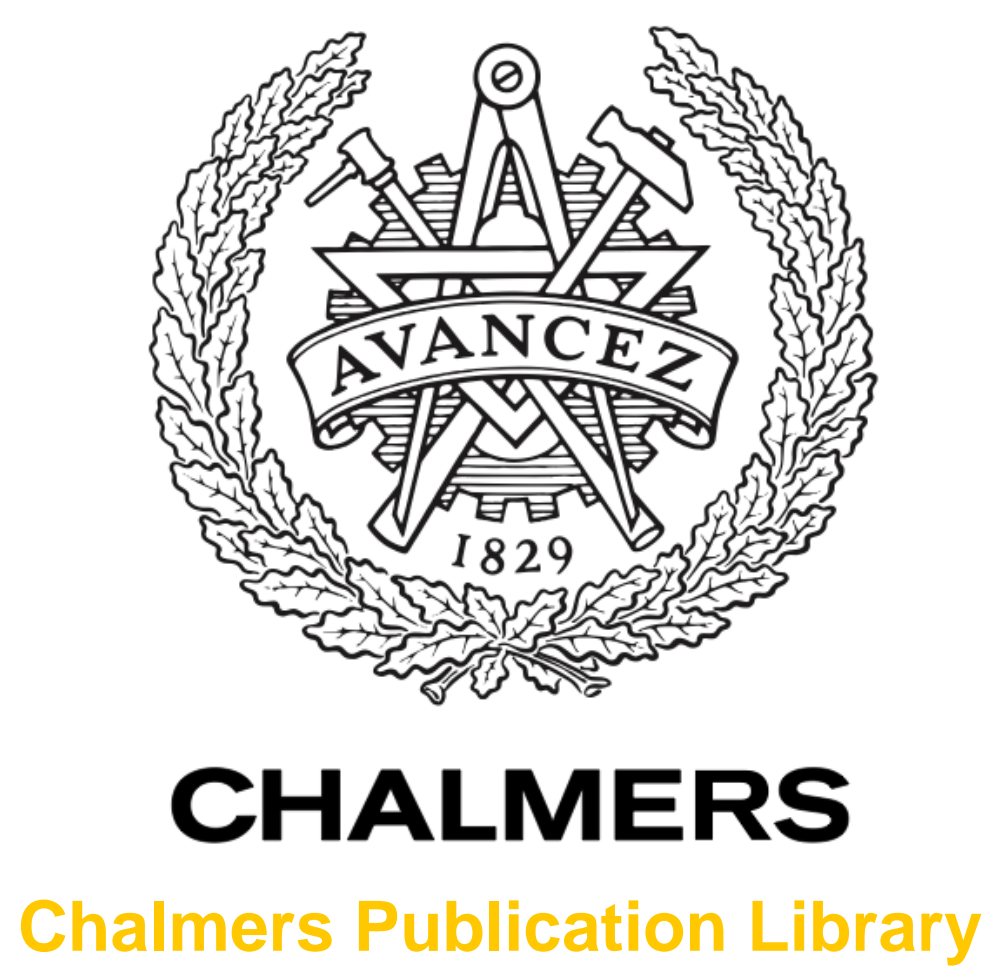

Robust Link Scheduling with Channel Estimation and Location Information

This document has been downloaded from Chalmers Publication Library (CPL). It is the author's version of a work that was accepted for publication in:

47th Asilomar conference on Signals, Systems and Computers

Citation for the published paper:

Muppirisetty, L. ; Di Taranto, R. ; Wymeersch, H. (2014) "Robust Link Scheduling with Channel Estimation and Location Information". 47th Asilomar conference on Signals,

Systems and Computers

Downloaded from: http://publications.lib.chalmers.se/publication/192645

Notice: Changes introduced as a result of publishing processes such as copy-editing and formatting may not be reflected in this document. For a definitive version of this work, please refer to the published source. Please note that access to the published version might require a subscription.

Chalmers Publication Library (CPL) offers the possibility of retrieving research publications produced at Chalmers University of Technology. It covers all types of publications: articles, dissertations, licentiate theses, masters theses, conference papers, reports etc. Since 2006 it is the official tool for Chalmers official publication statistics. To ensure that Chalmers research results are disseminated as widely as possible, an Open Access Policy has been adopted.

The CPL service is administrated and maintained by Chalmers Library. 


\title{
Robust Link Scheduling with Channel Estimation and Location Information
}

\author{
L. Srikar Muppirisetty, Rocco Di Taranto, and Henk Wymeersch \\ Department of Signals and Systems, Chalmers University of Technology, Gothenburg, Sweden \\ E-mail: \{srikar.muppirisetty, taranto, henkw\}@ chalmers.se
}

\begin{abstract}
We study the robust link scheduling problem (RLSP) based on a physical interference model with errors in channel state information. The objective of RLSP is to find a robust minimum length schedule using spatial time division multiple access. We compare two approaches to RLSP, one using channel gain estimates and the other using location information. In both cases, we formulate the RLSP as a binary integer program and solve it by a classical column generation technique. Our comparison reveals that both approaches yield similar performances, but with different overhead.
\end{abstract}

\section{INTRODUCTION}

Location-aware applications have gained prominence in recent years due to rapid advancements in positioning systems. Most wireless devices have the capability to know their geographic location based on systems such as global positioning system (GPS) [1], cellular-based positioning [2], and ultra-wide bandwidth (UWB) positioning [3]. Moreover, wireless devices in next-generation communication systems are expected to obtain location information with a very high level of accuracy, anywhere and anytime. Location information will play a key role in improving the communication capabilities of wireless applications at various layers of the protocol stack. Location information has been exploited in a variety of wireless applications in the literature, such as cognitive radio [4], relay selection and handover [5], and link rate adaptation [6]. In the network layer [7] demonstrates the use of position information for geographic routing. Location information has been used for scheduling in [8], [9] at the medium access control (MAC) layer.

Scheduling is a classical problem in the MAC layer and has been studied extensively. In traditional link scheduling, average channel gains are generally used to schedule links. A popular approach to link scheduling is spatial time division multiple access (STDMA) [10], which is a collisionfree scheme in which links are allocated time slots, and concurrent transmissions are allowed as long as they do not cause significant mutual interference [11]. The objective in STDMA link scheduling is to find the minimum number of time slots required to schedule all the links in the network. A mathematical programming formulation was introduced in [12] and modeled as a binary integer programming (BIP) problem, which was solved efficiently with column generation. The work in [13] extended [12] to robust scheduling, considering the stochastic nature of the links due to log-normal shadowing. Robust link scheduling was applied in [14] for vertical spectrum sharing in STDMA networks with traffic demand uncertainty. However, the framework in [12]-[14] is purely based on channel gains of the links in the network, leading to hidden node problems [15, Chapter 4]. Moreover, uncertainties that arise due to estimation of the channel gains (e.g., weak channels are harder to estimate than strong channels) were not considered.

In this paper, we study the centralized robust link scheduling problem, where channel state information is obtained either from beaconing with channel estimation (referred to a gain based scheduling, GBS) or from a positioning system (referred to as location based scheduling, LBS). We show that GBS and LBS have similar performance and exhibit similar behavior in terms of robustness to estimation errors. GBS is mainly limited by hidden node problems and poor scalability (in the worst case quadratic in the number of nodes). LBS has better scalability (linear in the number of nodes), does not suffer from hidden node problems, but is limited by mismatch due to shadowing.

Notation: $|E|$ denotes the cardinality of a set $E$; vectors are written in bold (e.g., $\mathbf{x}$ ), with $\mathbf{1}$ being the vector of all ones; $\mathbf{x} \preceq \mathbf{y}$ means that $x_{i} \leq y_{i}, \forall i$.

\section{System MOdeL}

We consider a wireless network of $N$ nodes, represented by a communication graph $G=(V, E)$ consisting of a set $V$ of vertices (nodes) and a given set $E \subseteq V \times V$ representing the the links between nodes, which are to be scheduled. Links will be scheduled when their signal-to-noise ratio (SNR) is sufficiently high, i.e., $(i, j) \in E$ if $\operatorname{SNR}_{i j}\left(g_{i j}\right) \geq \gamma$, in which $\operatorname{SNR}_{i j}\left(g_{i j}\right)=g_{i j} P / W$, where $g_{i j}$ is the channel gain between nodes $i$ and $j, P$ is the fixed power transmitted by node $i, W$ is the noise power at the receiver, and $\gamma$ is the target SNR requirement. The channel gain is modeled to comprise path loss and fading $g_{i j}=10^{-\mathrm{PL}\left(d_{i j}\right) / 10} 10^{Z / 10}$, where $Z$ is lognormal shadowing, $Z \sim \mathcal{N}\left(0, \sigma_{\mathrm{z}}^{2}\right), d_{i j}$ is the distance between the nodes $i$ and $j$, and the path loss is given by $\mathrm{PL}\left(d_{i j}\right)=$ $\mathrm{PL}\left(d_{0}\right)+10 \eta \log _{10}\left(d_{i j} / d_{0}\right)$, in which $\eta>0$ is the path loss exponent and $\mathrm{PL}\left(d_{0}\right)$ is the line-of-sight path loss at reference distance $d_{0}$.

Assuming a given STDMA schedule, where a subset $S=$ $S_{\mathrm{TX}} \times S_{\mathrm{RX}} \subseteq E$ of links are selected during a given time slot, each concurrent transmission must satisfy the signal-tointerference-plus-noise ratio (SINR) requirement

$$
\frac{g_{i j} P}{\sum_{m \in S_{\mathrm{TX}} \backslash\{i\}} g_{m j} P+W} \geq \gamma, \forall(i, j) \in S .
$$


In a real system, the actual channel gains $g_{i j}$ are generally not available, and instead the scheduler resorts to channel estimates to derive STDMA schedules. Due to the mismatch between assumed channel gain and actual channel gains, link outages will occur. Outages can be reduced by employing robust link scheduling.

\section{RobUSt LinK Scheduling}

In this section, we provide the mathematical formulation of the robust link scheduling problem (RLSP) [13].

\section{A. RLSP as an Integer Linear Program}

We introduce $x_{i j t} \in\{0,1\}$, with $x_{i j t}=1$ if time slot $t$ is assigned to link $(i, j) \in E$, and $y_{t} \in\{0,1\}$, where $y_{t}=1$ indicates that time slot $t$ is used. Let $\mathcal{T}$ be a feasible set of $T$ time slots, ${ }^{1}$ i.e., $\mathcal{T}=\{1,2,3, \ldots, T\}$. Following [13], the RLSP can be written as follows

$$
\begin{array}{ll}
\text { minimize } & \mathbf{y}^{\mathrm{T}} \mathbf{1} \\
\text { subject to } & \sum_{(i, j) \in E} x_{i j t} \leq y_{t}|E| \\
& \sum_{t \in \mathcal{T}} x_{i j t}=1 \\
& \operatorname{SINR}_{i j}(\tilde{\mathbf{g}}, \mathbf{x}) \geq \gamma \\
& \mathbf{g}^{-} \preceq \tilde{\mathbf{g}} \preceq \mathbf{g}^{+} \\
& \sum_{j:(i, j) \in E} x_{i j t}+\sum_{k:(k, i) \in E} x_{k i t} \leq 1 \\
& x_{i j t} \in\{0,1\}, y_{t} \in\{0,1\},
\end{array}
$$

where $\mathrm{g}^{-}$and $\mathrm{g}^{+}$are vectors of pessimistic and optimistic channel gains respectively, which will be discussed in Section IV, and

$$
\operatorname{SINR}_{i j}(\tilde{\mathbf{g}}, \mathbf{x})=\frac{\tilde{g}_{i j} x_{i j t} P+\left(1-x_{i j t}\right) M_{i j}}{\sum_{(m \neq i, n)} \tilde{g}_{m j} x_{m n t} P+W},
$$

where we tacitly assume that $\tilde{g}_{i j}=0$, when a link is not available to the scheduler. The scalar $M_{i j}$ is introduced to enable a BIP formulation ${ }^{2}$ of the RLSP.

We note the following: (2a) aims to minimize the total number of time slots required to schedule all the links in $E$; (2b) specifies that the number of links scheduled in one slot should not be more than the total number of links $|E|$; (2c) states that every link must be assigned a slot in the schedule; (2d) is the SINR requirement for successful transmission; (2e) states that the channel gains lie between ${ }^{3}$ pessimistic and optimistic values; (2f) states that a node cannot transmit and receive at the same time; and $(2 \mathrm{~g})$ imposes the integer requirements on the optimization variables.

Observe that links for which $\operatorname{SNR}_{i j}\left(g_{i j}^{-}\right)<\gamma$ are not feasible, so problem (2) is generally infeasible. These links

\footnotetext{
${ }^{1}$ As an example of a feasible set, $T=|E|$. To improve the convergence of the column generation method, we initialized RLSP with a basic feasible solution based on a heuristic mentioned in [12] .

${ }^{2}$ The constraint $(2 \mathrm{~d})$ can be satisfied when link $(i, j)$ is not assigned a time slot $t$ (i.e., $x_{i j t}=0$ ) by choosing $M_{i j}$ sufficiently large. For example [12] $M_{i j}=\gamma\left(\sum_{(m \neq i, n)} \tilde{g}_{m j} P+W\right)$.

${ }^{3}$ Observe that, equivalently, pessimistic gain values are used for transmitting link and optimistic gains are considered for interfering links in (2d).
}

can be removed from (2) to obtain a feasible problem and scheduled in a pure TDMA fashion. We will denote the number of removed links by $t_{\mathrm{T}}$. Note that due to hidden node problems, only a subset of channel gains are accounted in (2d) and (3).

We solved the RLSP using a column generation method by optimization package CVX [16] with mixed integer programming support from MOSEK [17].

\section{B. Performance Measures}

Let $\mathrm{y}^{*}$ and $\mathrm{x}^{*}$ be a solution to the RLSP, and the optimal value be $t_{\mathrm{S}}=\left(\mathbf{y}^{*}\right)^{\mathrm{T}} \mathbf{1}$. The length of the schedule is $t_{\mathrm{T}}+t_{\mathrm{S}}$, which we normalize with the number of links, so that

$$
t_{\text {norm }}=\mathbb{E}\left\{\frac{t_{\mathrm{T}}+t_{\mathrm{S}}}{|E|}\right\},
$$

is the expected normalized number of time slots, where the expectation is over shadowing realizations and position/channel estimates. As the actual channel gains are not known, certain scheduled links may not meet the SINR condition. We collect these links in a set $L$. The outage probability is defined as

$$
\begin{aligned}
P_{\text {out }} & =p\left(\operatorname{SINR}_{i j}\left(\mathbf{g}, \mathbf{x}^{*}\right)<\gamma\right) \\
& =\mathbb{E}\left\{\frac{|L|}{|E|}\right\} .
\end{aligned}
$$

Observe that (5) depends on the actual channel gains g. Note also that with complete (i.e., the channel gain is available for every pair of nodes) and exact (i.e., $\mathrm{g}^{-}=\mathrm{g}^{+}=\mathrm{g}$ ) channel gain information, $P_{\text {out }}=0$.

To capture the trade-off between schedule length and outage, we further introduce the normalized effective schedule length, which assumes that links in outage will be scheduled in TDMA fashion as

$$
\begin{aligned}
t_{\text {eff }} & =\mathbb{E}\left\{\frac{t_{\mathrm{T}}+t_{\mathrm{S}}}{|E|}+\frac{|L|}{|E|}\right\} \\
& =t_{\text {norm }}+P_{\text {out }} .
\end{aligned}
$$

\section{Pessimistic And Optimistic Channel Gains}

\section{A. General Approach and Motivation}

In this section, we describe two ways to obtain pessimistic $\left(\mathrm{g}^{-}\right)$and optimistic $\left(\mathrm{g}^{+}\right)$channel gain values. The first approach is based on direct channel estimation using beaconing signals, leads to the gain based scheduler (GBS). Provided enough beaconing resources are available, GBS can rely on accurate channel information, but has two drawbacks: (i) certain links may be too weak to estimate, thus leading to hidden node problems; (ii) for a network with $N$ nodes, $\mathcal{O}\left(N^{2}\right)$ channel gains may need to be estimated, which is prohibitive for large-scale networks. To mitigate these problems, our second approach to the RLSP is based on position information and leads to the location based scheduler (LBS). Here, the scheduler collects the locations of all the nodes, which scales as $\mathcal{O}(N)$, and computes $\mathrm{g}^{-}$and $\mathrm{g}^{+}$for every pair of nodes.

To have a consistent way to compare LBS and GBS, we introduce a robustness parameter $q \in[0,1]$, such that

$$
p\left(g_{i j}^{-} \leq g_{i j} \leq g_{i j}^{+} \mid \text {observation }\right)=q,
$$


where the "observation" may be either a channel estimate or the positions of nodes $i$ and $j$, as well as any available side information. When $q=0$, there is no robustness and we revert to a traditional non-robust STDMA scheduler; when $q=1$, all links in (2d) become infeasible, hence will be scheduled in TDMA, which is maximally robust.

\section{B. Robustness to Channel Estimation Uncertainty}

We consider estimating a single link $(i, j)$ and drop the subscripts for legibility. We assume nodes use training signals s of $N_{\text {tr }}$ unit-energy symbols to aid channel estimation. Channel estimation is only possible for links for which the SNR exceeds the so-called sensing threshold $\gamma_{\text {sense }} \leq \gamma$. The vector of received samples is $\mathbf{r}=h \mathbf{s}+\mathbf{w}$, where $h$ is the scalar complex flat fading channel such that $g=|h|^{2}$, and $w \sim \mathcal{C N}(0, W)$. Assuming no a priori channel information, the maximum likelihood estimate of $h$ is computed as $\hat{h}=\left(\mathbf{s}^{\mathrm{H}} \mathbf{s}\right)^{-1} \mathbf{s}^{\mathrm{H}} \mathbf{r}$, where $p(\hat{h} \mid h)=\mathcal{C N}\left(h, W / N_{\mathrm{tr}}\right)$ [18]. If we set $\hat{g}=|\hat{h}|^{2}$, we find that $\hat{g}$ has a non-central chi-squared distribution with two degrees of freedom [19]

$$
p(\hat{g} \mid g)=\frac{N_{\mathrm{tr}}}{W} \exp \left(-\frac{g+\hat{g}}{W / N_{\mathrm{tr}}}\right) I_{0}\left(\frac{\sqrt{g \hat{g}}}{\frac{1}{2} W / N_{\mathrm{tr}}}\right), \hat{g}>0,
$$

where $I_{0}(\cdot)$ is the zeroth order modified Bessel function of the first kind. Assuming a uniform a priori distribution $p(g)$, $p(g \mid \hat{g}) \propto p(\hat{g} \mid g)$, we can find $g^{-}$and $g^{+}$as solutions to

$$
\int_{g^{-}}^{g^{+}} p(g \mid \hat{g}) \mathrm{d} g=q .
$$

The posterior distribution $p(g \mid \hat{g})$ turns out to be asymmetric, so it may not always be possible to find the conservative gains to be symmetric around the estimated gain value. However, we can find them asymmetrically around $\hat{g}$ (i.e., $g^{-} \leq \hat{g} \leq g^{+}$) by numerically evaluating the integral (11).

\section{Robustness to Position Estimation Uncertainty}

Using positioning systems, each node can localize itself with an accuracy of $\sigma_{\text {pos }}$, expressed in meters. Let $d$ be the true distance between two nodes and $\hat{d}$ be its estimate. Assuming a Gaussian error [20] on the estimated distance, $p(\hat{d} \mid d)=\mathcal{N}\left(d, \sigma_{\mathrm{d}}^{2}\right)$, where $\sigma_{\mathrm{d}}=2 \sigma_{\text {pos }}$. Under a uniform a priori distribution, the posterior distribution is still Gaussian and is represented as

$$
p(d \mid \hat{d})=\frac{1}{\sqrt{2 \pi \sigma_{\mathrm{d}}^{2}}} \exp \left(-\frac{1}{2} \frac{(d-\hat{d})^{2}}{\sigma_{\mathrm{d}}^{2}}\right),
$$

and the pessimistic $d^{-}$and optimistic $d^{+}$distance values are found as the solutions to

$$
\int_{d^{-}}^{d^{+}} p(d \mid \hat{d}) \mathrm{d} d=q
$$

In this case the posterior distribution is Gaussian and symmetric, so it is possible to find conservative values that are symmetric around the estimate.

The pessimistic and optimistic channel gains which are needed for RLSP can be derived from the optimistic and pessimistic distances respectively, through the simple path

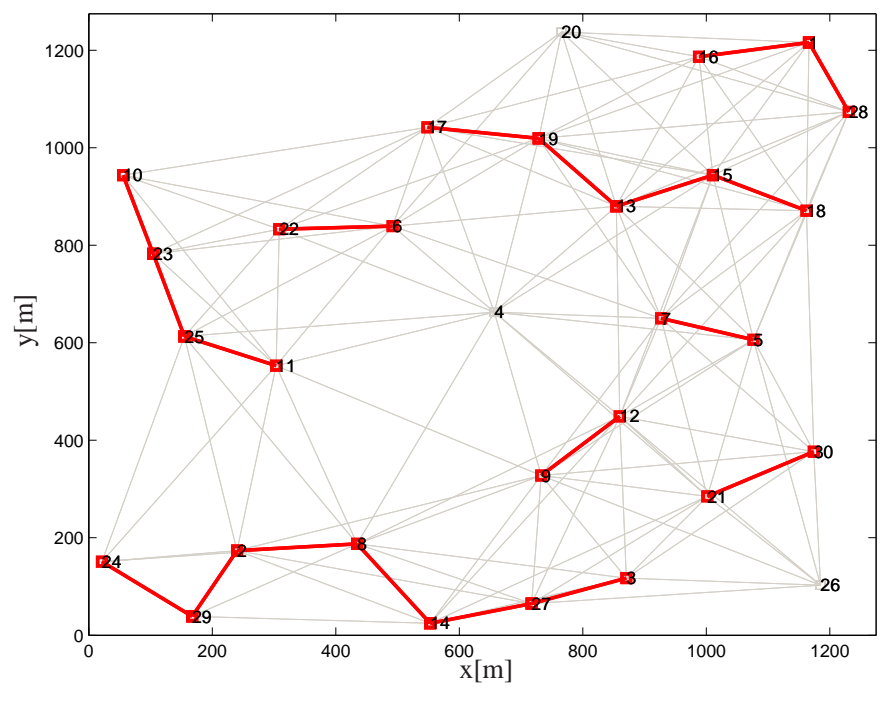

Figure 1. Random network topology with 30 nodes and 38 bidirectional links (marked in red). The grey shaded links correspond to the links that can be sensed based on a threshold $\gamma_{\text {sense }}=0 \mathrm{~dB}$.

Table I

SiMUlation PARAMETERS

\begin{tabular}{|c|c|c|c|}
\hline Parameter & Value & Parameter & Value \\
\hline \hline$\eta$ & 2.5 & $N_{\mathrm{tr}}$ & $5,200,3000$ symbols \\
\hline$P$ & $10 \mathrm{~W}$ & $\sigma_{\mathrm{pos}}$ & $0.5 \mathrm{~m}, 5 \mathrm{~m}, 50 \mathrm{~m}$ \\
\hline$\gamma$ & $10 \mathrm{~dB}$ & $q$ & {$[0,0.1, \ldots, 0.9]$} \\
\hline$\gamma_{\text {sense }}$ & $-6.5 \mathrm{~dB}$ & $W$ & $-70 \mathrm{dBm}$ \\
\hline$d_{0}$ & $1 \mathrm{~m}$ & $\sigma_{\mathrm{z}}$ & 2 \\
\hline
\end{tabular}

loss model. ${ }^{4}$ The pessimistic channel gain is then $g^{-}=$ $10^{-\mathrm{PL}\left(d^{+}\right) / 10}$ and the optimistic channel gain is $g^{+}=$ $10^{-\mathrm{PL}\left(d^{-}\right) / 10}$.

\section{RESUlts AND Discussion}

In this section, we show results on normalized minimum schedule length $t_{\text {norm }}$, outage probability $P_{\text {out }}$, and normalized effective schedule length $t_{\text {eff }}$ of the GBS and LBS.

\section{A. Simulation Setup}

We consider a random network with 30 nodes and 38 links in a square area of $1250 \mathrm{~m} \times 1250 \mathrm{~m}$ as shown in Fig 1 . The simulation parameters used to obtain the numerical results are given in Table I. For each value of $q, 50$ realizations of the shadowing channel are obtained and for every realization, 50 Monte Carlo experiments are performed to calculate $t_{\text {norm }}$ and $P_{\text {out }}$. The number of training symbols $N_{\mathrm{tr}} \in\{3000,200,5\}$ in GBS are chosen so that the channel estimation performance ranges from good over medium to poor. The position uncertainties $\sigma_{\text {pos }} \in\{0.5 \mathrm{~m}, 5 \mathrm{~m}, 50 \mathrm{~m}\}$ used in LBS correspond to uncertainties attained by UWB, GPS, and cellular positioning systems respectively.

\section{B. Discussion}

Impact of Sensing Threshold: We first quantify the behavior of performance metrics $t_{\text {norm }}, t_{\text {eff }}$, and $P_{\text {out }}$ with actual channel gains as a function of sensing threshold $\gamma_{\text {sense }}$ for

\footnotetext{
[13]. 

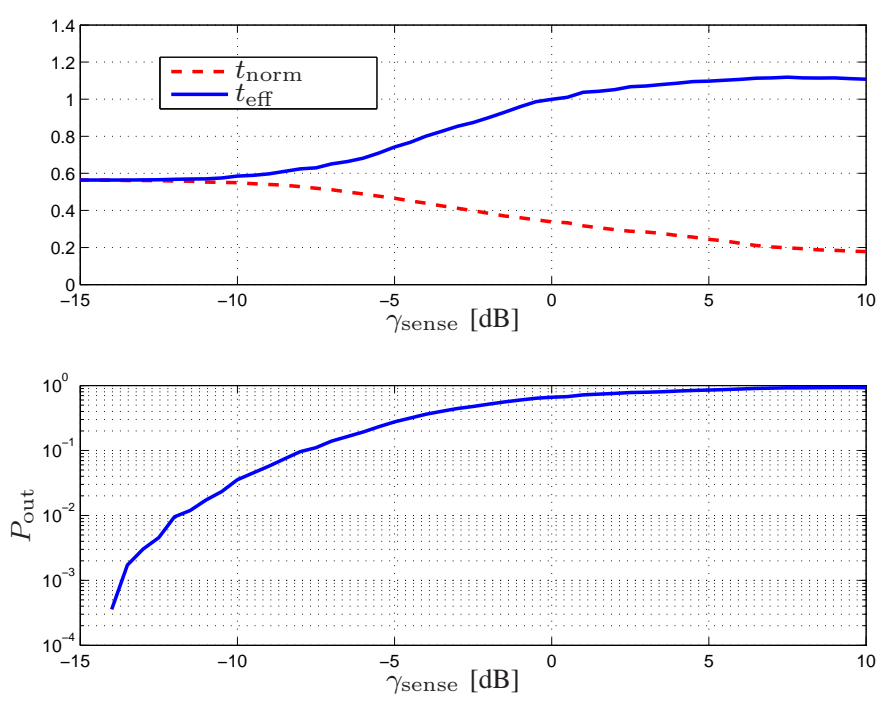

Figure 2. Schedule length, effective schedule length, and outage probability with actual channel gains for GBS as a function of the sensing threshold $\gamma_{\text {sense. }}$

GBS (see Fig. 2). If the sensing threshold $\gamma_{\text {sense }}$ is decreased, then $t_{\text {norm }}$ will increase due to the additional information of more interfering links. In turn, this increase in $t_{\text {norm }}$ translates in a decrease in $P_{\text {out }}$, as the scheduler becomes more aware of the entire network. It can be observed in Fig. 2 for $\gamma_{\text {sense }} \leq-14.5 \mathrm{~dB}$, the network becomes fully connected, thus $t_{\text {eff }}=t_{\text {norm }}$ and $P_{\text {out }} \rightarrow 0$. Conversely, as $\gamma_{\text {sense }} \rightarrow \gamma$, $P_{\text {out }}$ rapidly increases, due to the limited knowledge about interfering links available to the scheduler. In the remainder of this section, we set $\gamma_{\text {sense }}$ to $-6.5 \mathrm{~dB}$, corresponding to $P_{\text {out }} \approx 0.1$.

Schedule Length: Fig. 3 shows $t_{\text {norm }}$ for both GBS and LBS as a function of $q$. It can be observed that $t_{\text {norm }}$ with actual channel gains in GBS is lower than $t_{\text {norm }}$ with actual distances of LBS. GBS has limited knowledge of the interfering links based on sensing threshold $\gamma_{\text {sense, }}$, so it underestimates interference and thus needs fewer slots to schedule the links. Moreover $t_{\text {norm }}$ is independent of $q$, because there is no robustness when the actual values are known.

Now consider the case when there is uncertainty, say $N_{\mathrm{tr}}=200$ for GBS. It can be seen from Fig. 3 that $t_{\text {norm }}$ increases with larger value of $q$, since an increase in $q$ leads to a decrease in $g_{i j}^{-}$, so that more links will not satisfy $\operatorname{SNR}_{i j}\left(g_{i j}^{-}\right) \geq \gamma$. These links are scheduled in TDMA, hence $t_{\mathrm{T}}$ increases. At the same time, $t_{\mathrm{S}}$ will also increase due to the use of conservative channel gains in the SINR constraint. By similar arguments, $t_{\text {norm }}$ for LBS will also increase with $q$, for any case of uncertainty value $\sigma_{\text {pos }}$. Finally, all the schedules will converge to a TDMA schedule when $q=1$.

Let us now analyze the behavior of $t_{\text {norm }}$ across different levels of uncertainty (i.e., different values of $N_{\mathrm{tr}}$ for GBS or of $\sigma_{\text {pos }}$ for LBS). Note that the shape of the posterior distribution $p(d \mid \hat{d})(12)$ or $p(g \mid \hat{g})$ (10) is narrow with low uncertainty and broad with high uncertainty. Hence, for a particular value of $q$, the pessimistic value decreases and the optimistic value increases quickly with higher uncertainty in the estimate. As a

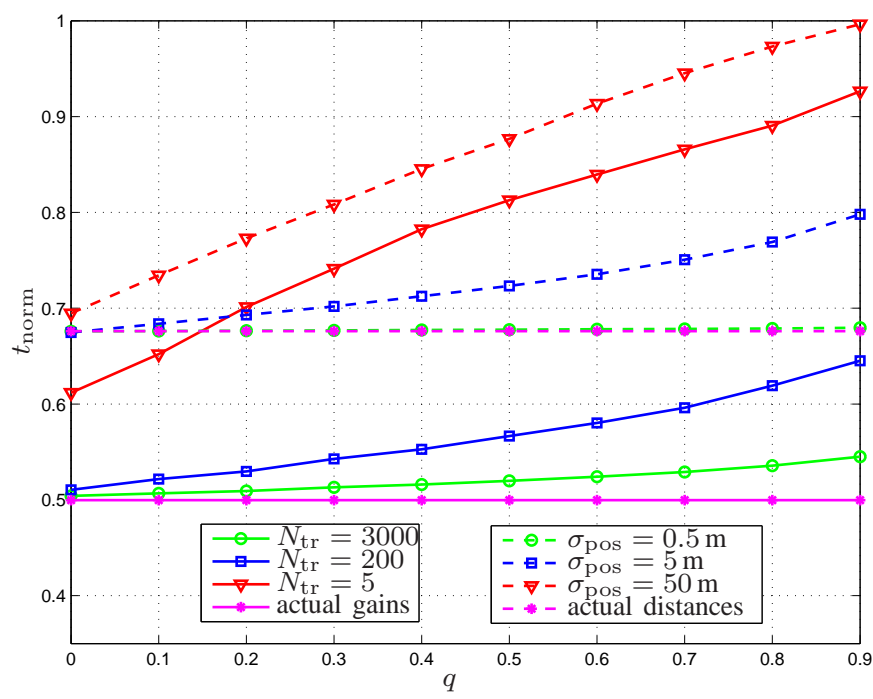

Figure 3. Normalized minimum length schedule for GBS (solid) and LBS (dashed) as a function of the robustness parameter $q$.

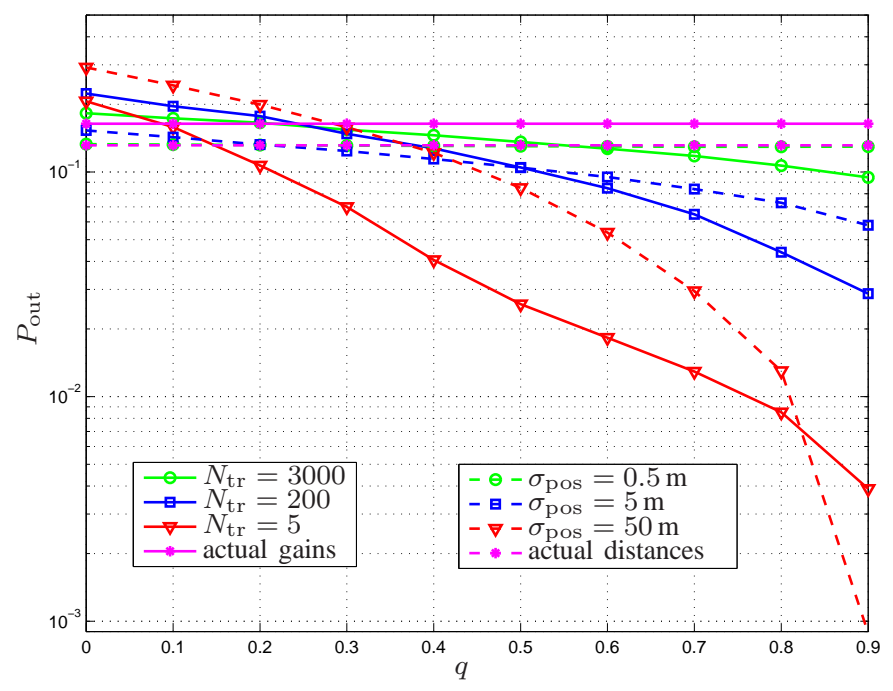

Figure 4. Outage performance of for GBS (solid) and LBS (dashed) as a function of the robustness parameter $q$.

result $t_{\text {norm }}$ is larger when the uncertainty in the estimates is higher. This can be easily noticed in Fig. 3, where, for example for LBS, $t_{\text {norm }}$ with $\sigma_{\text {pos }}=50 \mathrm{~m}$ is always larger than with $\sigma_{\text {pos }}=5 \mathrm{~m}$, for any value of $q$.

Outage: Fig. 4 shows the outage performance $P_{\text {out }}$ for both schedulers. Note that GBS, which generally has lower $t_{\text {norm }}$ than LBS leads to more outages due to hidden node problems. These outages can be mitigated by reducing $\gamma_{\text {sense }}$ at a cost of higher receiver sensitivity. In LBS outages occur even when there is no position uncertainty, due to the mismatch between the assumed channel gain (without shadowing) and actual channel gain (with shadowing). Any additional mismatch (e.g., between the actual and assumed path loss coefficient $\eta$ ) will cause additional outages.

With position uncertainty or channel estimation uncertainty, outages decrease when $q$ increases. The reason is that we are more conservative and allocate more time slots to schedule 


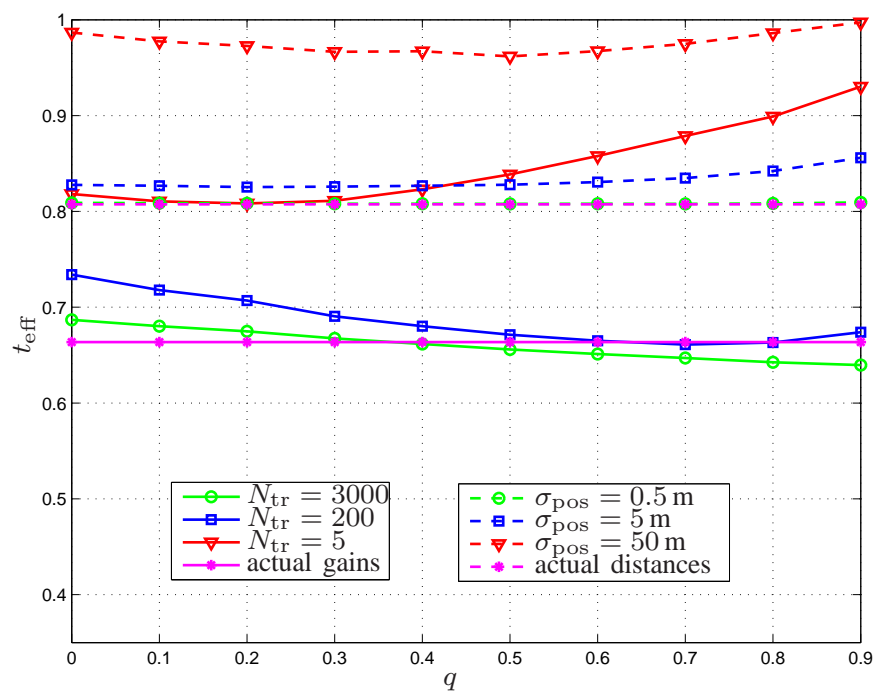

Figure 5. Effective schedule length for GBS (solid) and LBS (dashed) as a function of the robustness parameter $q$.

links, so outages are less likely to occur. For any value of $q$, the outage $P_{\text {out }}$ is larger with higher uncertainty, because the schedule is generated with values quite different from the actual values. The links that satisfy the SINR constraint when the schedule is made may not satisfy it with actual values. As $q$ increases, schedulers working with higher uncertainties will see a faster drop in $P_{\text {out }}$ (resulting in cross-over points in Fig. 4, e.g., beyond $q \approx 0.4$ LBS with $\sigma_{\text {pos }}$ has the smallest outage), at a cost in terms of the schedule length.

Effective Schedule Length: Fig. 5 shows, as a function of $q$, the normalized effective schedule length $t_{\text {eff }}$, which describes the trade-off between schedule length (as provided by the LBS or GBS) and the corresponding outages. We observe that for GBS (resp. LBS), $t_{\text {eff }}$ is around $17 \%$ (resp. 12\%) larger than $t_{\text {norm }}$ when using the actual channel gains (resp. distances). In the presence of uncertainty an increase in $q$ leads to an initial reduction in $t_{\text {eff }}$ due to a reduction in outages. Further increase in $q$ lead to an increase in $t_{\text {eff }}$, due to the dominating effect of an excessively long schedule. For example, in GBS, it can be noticed that there is an optimal robustness parameter $q \approx 0.2$ for $N_{\mathrm{tr}}=5$ and $q \approx 0.7$ for $N_{\mathrm{tr}}=200$. Interestingly, GBS tends to favor large values of $q$, while LBS prefers low values of $q$, indicating the LBS is inherently more robust. While overall, LBS is outperformed by GBS, the gap between the two will shrink when $\gamma_{\text {sense }}$ is increased. In particular, from Fig. 2, we can see that for $\gamma_{\text {sense }} \approx-4 \mathrm{~dB}$, GBS and LBS will lead to similar $t_{\text {eff }}$.

\section{CONCLUSIONS}

We studied the benefits and drawbacks of robust link scheduling based on channel estimates or on position estimates. We have demonstrated that link scheduling using channel gains suffered significant outage due to its limited sensing capability of interfering links, even when robustness is considered in the schedule. Such hidden node problems can be mitigated using location-based scheduling. Moreover, position information scales only linearly with number of nodes, where as channel state information scales quadratically. However, the performance of location-based scheduling is limited by the amount of shadowing in the channel.

The location-based scheduler performance can be improved by taking into consideration the uncertainty of shadowing. Furthermore, the performance can be enhanced when there is an availability of partial channel state information, which can be exploited using spatial correlation models.

\section{ACKNOWLEDGMENT}

This research was supported in part, by the European Research Council, under Grant No. 258418 (COOPNET). The authors would like to thank Johan Winges for the support related to numerical issues and MOSEK for the free academic license to use their numerical optimization software.

\section{REFERENCES}

[1] M. S. Grewal, L. R. Weill, and A. P. Andrews, Global positioning systems, inertial navigation, and integration. Wiley-Interscience, 2007.

[2] B. Ludden and L. Lopes, "Cellular based location technologies for UMTS: a comparison between IPDL and TA-IPDL," vol. 2, pp. 13481353,2000

[3] S. Gezici, Z. Tian, G. B. Giannakis, H. Kobayashi, A. F. Molisch, V. H. Poor, and Z. Sahinoglu, "Localization via ultra-wideband radios," IEEE Signal Processing Magazine, pp. 70-84, 2005.

[4] H. Celebi and H. Arslan, "Utilization of location information in cognitive wireless networks," IEEE Wireless Communications, vol. 14, no. 4, pp. 6-13, Aug. 2007.

[5] J. H. Yap, X. Yang, S. Ghaheri-Niri, and R. Tafazolli, "Position assisted relaying and handover in hybrid ad hoc WCDMA cellular system," IEEE International Symposium on Personal, Indoor and Mobile Radio Communications, 2002.

[6] S. Sand, R. Tanbourgi, C. Mensing, and R. Raulefs, "Position aware adaptive communication systems," Proceedings of the 43rd Asilomar conference on Signals, systems and computers, pp. 73-77, 2009.

[7] D. Chen and P. K. Varshney, "Geographic routing in wireless ad hoc networks," Guide to Wireless Ad Hoc Networks, pp. 151-188, 2009.

[8] W. Ning and B. Randall, "Location-based MAC protocols for mobile wireless networks," Information Theory and Applications Workshop, 2007.

[9] S. Min-Te, H. Lifei, A. Anish, and L. Ten-Hwang, "Location-based MAC protocols for mobile wireless networks," Wireless Communications and Mobile Computing, 2003.

[10] P. Djukic and S. Valaee, "Link scheduling for minimum delay in spatial re-use TDMA," INFOCOM, pp. 28-36, 2007.

[11] R. Nelson and L. Kleinrock, "Spatial TDMA: A collision free multihop channel access protocol," IEEE Transactions on Communications, vol. 33, no. 9, pp. 934-944, Sep 1985.

[12] P. Värbrand, D. Yuan, and P. Björklund, "Resource optimization of spatial TDMA in ad hoc radio networks: a column generation approach," INFOCOM, 2003.

[13] K. Papadaki and V. Friderikos, "Robust scheduling in spatial reuse TDMA wireless networks," IEEE Transactions on Wireless Communication, vol. 7, no. 12, pp. 4767-4771, 2008.

[14] P. Phunchongharn, E. Hossain, L. B. Le, and S. Camorlinga, "Robust scheduling and power control for vertical spectrum sharing in STDMA wireless networks," IEEE Transactions on Wireless Communication, vol. 11, no. 5, May 2012.

[15] A. S. Tanenbaum and D. J. Wetherall, Computer Networks, 5th ed. Prentice Hall, 2011.

[16] CVX Research, Inc., "CVX: Matlab software for disciplined convex programming, version 2.0 beta," http://cvxr.com/cvx, Sep. 2012.

[17] "The MOSEK optimization software." [Online]. Available: http://www. mosek.com/

[18] S. M. Kay, Fundamentals of statistical signal processing: estimation theory. Upper Saddle River, NJ, USA: Prentice-Hall, Inc., 1993.

[19] J. G. Proakis, Digital Communications. McGraw-Hill, 1995.

[20] M. Haenggi, "Link modeling with joint fading and distance uncertainty," 4th International Symposium on Modeling and Optimization in Mobile, Ad-Hoc and Wireless Networks, Apr. 2006. 